\title{
Preliminary Attempt to Develop a Path-Flow Analysis Model for Swimming Performance in Children
}

\author{
Joel Coelho ${ }^{1}$, Ana M Cruz ${ }^{1,4}$, Daniel A Marinho ${ }^{2,4}$, Mário C Marques ${ }^{2,4}$, Aldo M Costa ${ }^{2,4}$, \\ António J Silva ${ }^{3,4, *}$ and Tiago M Barbosa ${ }^{1,4}$ \\ ${ }^{1}$ Polytechnic Institute of Bragança, Bragança, Portugal \\ ${ }^{2}$ University of Beira Interior, Covilhã, Portugal \\ ${ }^{3}$ University of Trás-os-Montes and Alto Douro, Vila Real, Portugal \\ ${ }^{4}$ Research Centre in Sports, Health and Human Development, Vila Real, Portugal
}

\begin{abstract}
The aim of this research was to develop a path-flow analysis model from age-group swimmer's performance based on biomechanical and energetic parameters. Twenty two young male age-group swimmers with several competitive levels volunteered to serve as subjects. It was assessed swim velocity, stroke length, stroke frequency, stroke index, propulsive efficiency, critical velocity and $200 \mathrm{~m}$ freestyle event performance. To verify the quality of the model, root mean square residuals (RMSR) was computed. The confirmatory model explained $54 \%$ of swimming performance. RMSR was 0.064. As a conclusion, the model based in biomechanical and energetic variables, according to the relationships suggested is appropriated to explain performance in age-group swimmers.
\end{abstract}

Keywords: Swimming, young swimmers, performance, biomechanics, energetics.

\section{INTRODUCTION}

The goal of competitive swimming is to travel the event distance as fast as possible. The identification of the parameters that predict swimming performances is one of the main aims of the swimming "science" community. Indeed, it is consensual that biomechanical and energetic variables are determinant for performance in this sport. Several research groups dedicate their attention to the relationships establish between biomechanical and energetic variables on adult swimmers, with special emphasis on elite adult swimmers. However, several parameters often assessed in adult swimmers are not able to be used in age-groups due to several reasons. Even so, on regular basis, age-group coaches also do biomechanical and energetic assessments but that are less expensive, evasive or complex. Nevertheless, the understanding of the relationships establish between these domains in age-group swimming is not fully understood (e.g. [1]).

The aim of this research was to develop a path-flow analysis model from age-group swimmer's performance based on biomechanical and energetic parameters. The theoretical model was developed according to main review papers about these relationships (e.g. [2]) and the age-group coach's assessments, being presented in Fig. (1).

*Address correspondence to this author at the Department of Sport Sciences, University of Trás-os-Montes and Alto Douro, Vila Real, Portugal, Rua Dr. Manuel Cardona, 5000 Vila Real Portugal; Tel: +351 259350000; E-mail: Ajsilva@utad.pt

\section{METHODS}

\section{Subjects}

Twenty two male age-group swimmers with several competitive levels volunteered to serve as subjects (12.67 \pm 0.49 years old; $1.56 \pm 0.08 \mathrm{~m}$ of height; $46.08 \pm 7.14$ $\mathrm{kg}$ of body mass; $16.38 \pm 5.77 \%$ fat mass; Tanner stages 1-2). Parents and coaches gave their consent for the swimmers participate in this study. All procedures were in accordance to the Declaration of Helsinki in respect to Human research.

\section{Data Collection}

Each swimmer made a maximal $25-\mathrm{m}$ swim with an underwater start. The swimmers were advised to reduce gliding during the start. Swimming velocity was measured in the middle $15-\mathrm{m}$ as:

$$
\overline{\mathrm{v}}=\frac{\mathrm{d}}{\mathrm{t}}
$$

Where $v$ is the mean swimming velocity, $d$ the distance covered by the swimmer, $t$ the time spent to cover such distance and measured with a chronometer by an expert evaluator. The stroke frequency (SF) was measured with a crono-frequency meter from 3 consecutive stroke cycles, in the middle of the $15-\mathrm{m}$ distance by an expert evaluator as well. Stroke length was estimated as [3]:

$$
\mathrm{SL}=\frac{\overline{\mathrm{v}}}{\mathrm{SF}}
$$




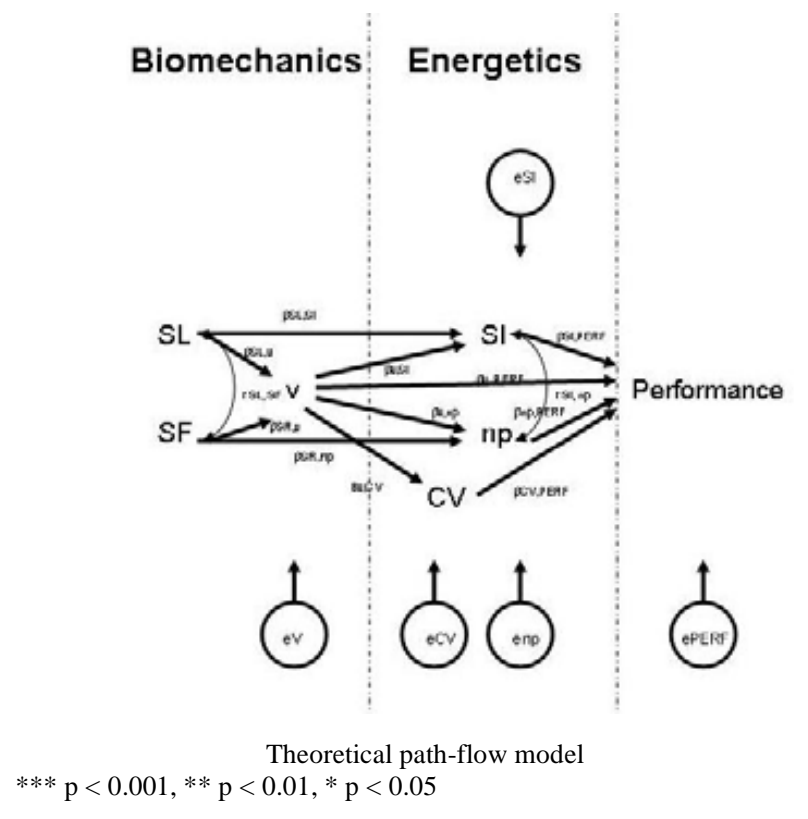

Fig. (1). Theoretical and confirmatory path-flow models.

Stroke index (SI) considered as a swim stroke efficiency index was computed [4]:

$$
S I=S L \cdot \bar{v}
$$

Propulsive efficiency ( $\eta$ ) was also estimated [5]:

$$
\eta_{p}=\left(\frac{v \cdot 0.9}{2 \pi \cdot S F \cdot l}\right) \cdot \frac{2}{\pi}
$$

Where $v$ is the swim velocity, $S F$ is the stroke frequency and $l$ is the arm's length.

Critical velocity (CV) was computed based on the swimmers curriculum [6] on the $200 \mathrm{~m}$ and $800 \mathrm{~m}$ freestyle short course events. The $C V$ was calculated using the slope of the simple linear regression model, plotting the swimming performances over the time and determined by:

$$
d=a . t+b
$$

Where $d$ is the distance of the swim event, $a$ is the slope of the fit line, $t$ is the time spent to cover the distance and $b$ the $y$ - interception in the origin of the $x x$ axis.

Swimming performance was assessed by the $200 \mathrm{~m}$ freestyle event in official short course competition. Time gap between biomechanical plus energetic assessments and swim performance were made in no more than two weeks.

\section{Statistical Procedures}

The normality of the distributions was evaluated with the Shapiro-Wilk test. Descriptive statistics (mean $\pm 1 \mathrm{SD}$ ) from all variables were calculated.

Path-flow analysis was performed with the estimation of linear regression standardized coefficients between the exogenous and endogenous variables. All assumptions to per-

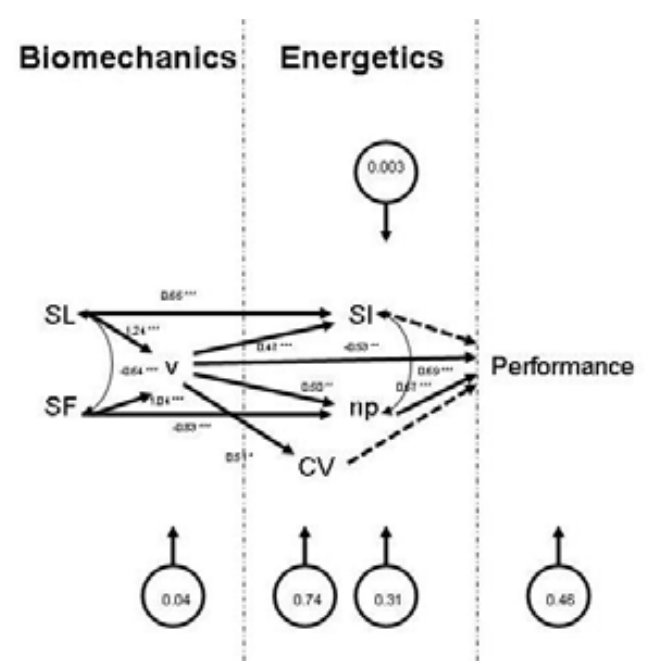

Confirmatory path-flow model

form the path-flow analysis were taken into account. When appropriate, according to the theoretical model, simple or multiple linear regression models were computed. Standardized regression coefficients $(\beta)$ were considered. Significance of each $\beta$ was assessed with the t-Student test $(p<0.05)$. The effect size of the disturbance term, reflecting unmeasured variables, for a given endogenous variable, was $1-\mathrm{R}^{2}$.

To verify the quality of the model, root mean square residuals (RMSR) was computed:

$R M S R=\sqrt{\frac{\sum_{i=1}^{p} \sum_{i=1}^{q}(r i j-p i j)^{2}}{p+q}}$

Where $r$ is the Pearson correlation coefficients and $p$ the correlation predicted by the model (based in total effect, i.e., the addiction of the direct and indirect effects plus spurious effects). Qualitatively, it is considered that if: (i) RMSR < 0.1 the model adjust to the theory; (ii) RMSR $<0.05$ the model adjusts very well to the theory and; (iii) RMSR $\sim 0$ the model is perfect.

\section{RESULTS AND DISCUSSION}

Table 1 presents descriptive statistics from all variables studied. Mean data values are somewhat within the range of values reported in the literature for swimmers with similar chronological age and gender (e.g. [7-9]). Data dispersion, expressed as $1 S D$, was moderate-high for almost every variable. This same idea can be supported analyzing the range values.

Fig. (1) presents the theoretical and confirmatory pathflows for age-group swimmer's performance. Almost every partial relationship confirmed the hypothesis. The only ex- 
Table 1. Descriptive Statistics of Biomechanical Variables, Energetic Variables and Swim Performance

\begin{tabular}{|c|c|c|c|c|}
\hline & Mean & Standard Deviation & Min & Max \\
\hline \hline $\mathrm{SL}(\mathrm{m})$ & 1.62 & 0.19 & 0.25 & 1.14 \\
\hline $\mathrm{SF}(\mathrm{Hz})$ & 0.90 & 0.08 & 1.20 & 1.69 \\
\hline $\mathrm{v}\left(\mathrm{m} \cdot \mathrm{s}^{-1}\right)$ & 1.45 & 0.11 & 1.60 & 3.16 \\
\hline $\mathrm{SI}\left(\mathrm{m}^{2} \cdot \mathrm{c}^{-1} \cdot \mathrm{s}^{-1}\right)$ & 2.37 & 0.40 & 17.27 & 34.93 \\
\hline $\mathrm{np}(\%)$ & 24.07 & 5.25 & 0.87 & 1.26 \\
\hline $\mathrm{CV}\left(\mathrm{m} \cdot \mathrm{s}^{-1}\right)$ & 1.08 & 0.11 & 136.12 & 206.27 \\
\hline $200 \mathrm{~m}$ freestyle $(\mathrm{s})$ & 157.20 & 17.41 & & \\
\hline
\end{tabular}

ception was the relationship between $\eta p$ and performance $(\beta=0.67)$, as it is considered in the literature that best performances are achieved in most efficient subjects [5]. $C V$ and $S I$ did not include the final model as t-Student test was not-significant. New studies should focus in these phenomenons to clear out data reported here. The confirmatory model explained $54 \%$ of swimming performance. So, the correlation coefficient was 0.73 , meaning qualitatively a high prediction. RMSR was 0.064. In this sense, the confirmatory path-flow model can be considered suitable of the theory presented. Although the model is appropriate, it do not explains $46 \%$ of the swim performance. Swimming performance is a multifactorial phenomenon, where e.g. psychological, anthropometrical, genetic, motor control or environmental aspects also play a significant role (e.g. [10]) and were not included in this model. Moreover, there are as well biomechanical and energetic variables that were not inserted, such as speed fluctuation, segmental velocities', energy cost or energy expenditure [2] since they are less suitable to be used with age-group swimmers.

As a conclusion, the model based in biomechanical and energetic variables, according to the relationships suggested is appropriated to explain performance in age-group swimmers. Nevertheless, the model should be expanded, including other variables in order to increase the prediction level of the swimming performance.

\section{REFERENCES}

[1] Jurimae J, Haljaste K, Cichella A, et al. Analysis of swimming performance from physical, physiological and biomechanical parameters in young swimmers. Pediatr Exerc Sci 2007; 19: 70-81.

[2] Barbosa TM, Bragada JA, Reis VM, et al. Energetics and biomechanics as determining factors of swimming performance: updating the state of the art. J Sci Med Sports 2009; (in press).

[3] Craig A, Pendergast D. Relationships of stroke rate, distance per stroke and velocity in competitive swimming. Med Sci Sports Exerc 1979; 11: 278-83.

[4] Costill D, Kovaleski J, Porter D, et al. Energy expenditure during front crawl swimming: predicting success in middle-distance events. Int J Sports Med 1985; 6: 266-70.

[5] Zamparo P, Pendergast D, Mollendorf J, Termin A, Minetti A. An energy balance of front crawl. Eur J Appl Physiol 2005; 94: 134-44.

[6] Costa AM, Silva AJ, Louro H, et al. Can the curriculum be used to estimate critical velocity in young competitive swimmers? J Sports Sci Med 2009; 8: 17-23.

[7] Greco CC, Denadai B. Critical speed and endurance capacity in young swimmers: effects of gender and age. Pediatric Exerc Sci 2005; 17: 353-63.

[8] Reis J, Alves F. Training induced changes in critical velocity and V4 in age group swimmers. In: Vilas-Boas JP, Alves F, Marques A, Eds. Biomechanics and medicine in swimming X; Portuguese J. Sport Sci 2006; pp.311-13.

[9] Schidt A, Ungerechts BE. The effect of cognitive intervention on stroke distance in age-group swimmers. In: Nomura T, Ungerechts BE, Eds. The Book of Proceedings of the 1st International Scientific Conference of Aquatic Space Activities; 2008: University of Tskuba, Tsubuka; 2008; pp. 380-7.

[10] Lavoie JM, Montpetit R. Applied physiology of swimming. Sports Med 1986; 3: 165-88. 